\title{
Centro de Informações e Biblioteca em Educação: estrutura e funcionamento
}

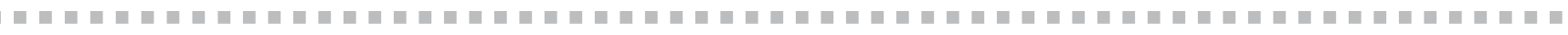
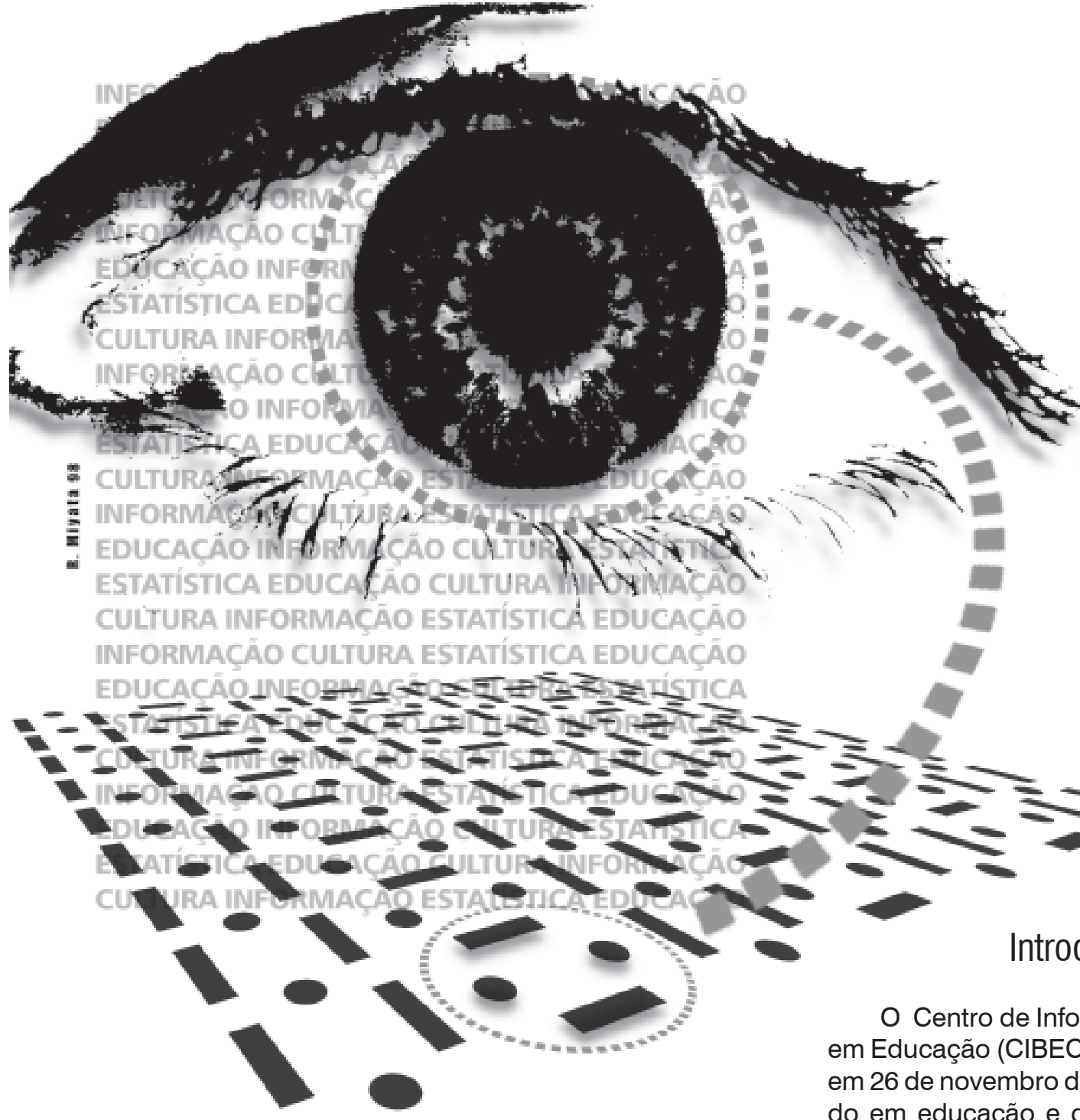

(1)

CULTURASUEAI

ESTATISTICA EDUCA EÁO CULTURA hIAOYMACÁC

CULTURA INFORIMACÃO ESTATISTICÁ EDUCACĀO

INFORIMACÄO CULTURA ESTATISTICA EDUCACÃO

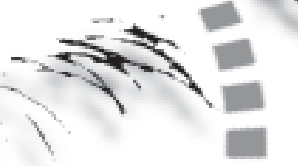

-

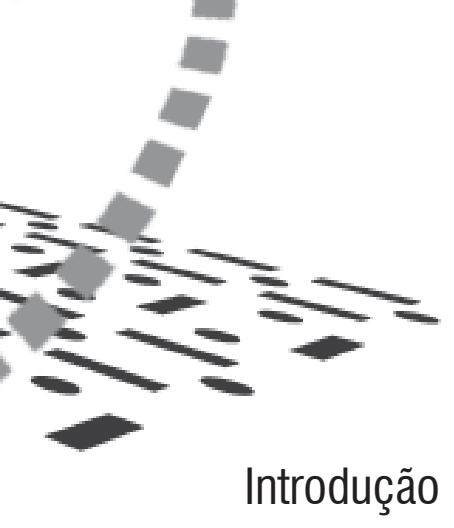

O Centro de Informações e Biblioteca em Educação (CIBEC), a ser reinaugurado em 26 de novembro de 1998, é especializado em educação e disponibiliza informações sobre avaliação e estatísticas educacionais, produzidas pelo próprio Instituto de Estudos e Pesquisas Educacionais (INEP), e outras, de instituições nacionais e internacionais.

A concepção do CIBEC tem por base as mudanças ocorridas na política educacional brasileira a partir de 1995. Estas 
mudanças vieram reforçar o processo de descentralização da gestão de políticas públicas, incrementando os canais de participação da sociedade civil e gerando maior articulação entre os tomadores de decisão, exigindo do Governo Federal um eficiente sistema de informação de acesso público.

O sistema de informações implantado pelo CIBEC permite a disseminação virtual e local, com foco no treinamento do usuário, seja ele institucional ou individual. Esta filosofia reflete os aspectos relativos à transparência das informações que subsidiam as decisões governamentais e a "prestação de contas" das ações de governo.

Este relato apresenta um breve histórico e detalha a atual estrutura e funcionamento do CIBEC, seus produtos e serviços.

\section{Breve histórico}

A idéia de uma biblioteca especializada em educação surgiu com a própria criação do INEP, em 13 de janeiro de 1937 (Lei no 378 ). Desde então, contribuiu para o desenvolvimento da educação no Brasil, tendo a importância e excelência do seu acervo sido reconhecidas pela UNESCO. Em 1976, com a transferência do INEP do Rio de Janeiro para Brasília, grande parte de seu acervo foi cedido à Universidade Federal do Rio de Janeiro (UFRJ). Instalada em Brasília, incorporou todo o material bibliográfico e arquivístico de várias bibliotecas do MEC, tais como a da Secretaria de Ensino Superior e a do Centro Brasileiro de Pesquisas Educacionais (CBPE).

Em 1981 foi criado o Centro de Informações Bibliográficas em Educação (CIBEC), pela Portaria Ministerial $n=612$, de 11 de novembro de 1981. O CIBEC tinha como objetivos básicos: (i) adquirir, processar e disseminar documentos na área educacional, em suporte convencial ou não, produzidos dentro e fora do país e; (ii) garantir a preservação da produção bibliográfica dos órgãos da administração direta e indireta do MEC, com exceção das publicações geradas pelas instituições de Ensino Superior e das Escolas Técnicas Federais - Portaria $\mathrm{n}$ - 263, de 14 de julho de 1982. Além disso, o CIBEC também deveria atuar como Unidade Central do Siste- ma de Informações Bibliográficas em Educação (SIBE).

O SIBE, também criado pela Portaria Ministerial $\mathrm{n}$ 0 612, era um complexo de bibliotecas e/ou centros de documentação brasileiros, organizados em forma de rede, que deveria desenvolver tarefas descentralizadas de coleta, aquisição de publicações nacionais e estrangeiras, tratamento cooperativo de informações e acesso às publicações, tendo como suporte uma base de dados em educação. Seu objetivo era fomentar o melhor aproveitamento da produção científica brasileira e dos recursos bibliográficos existentes nas unidades que o compunham. Com a base de dados do SIBE tinha-se a Bibliografia Brasileira de Educação (BBE).

A implementação do SIBE, entretanto, sofreu uma série de restrições, inclusive de ordem operacional e política, que inviabilizaram o seu funcionamento e acabaram por comprometer a execução das atividades propostas para o CIBEC. O Centro ficou, então, restrito ao cumprimento das funções mais tradicionais de uma biblioteca. Esse processo de desarticulação agravou-se, em 1986, com a interrupção das assinaturas de periódicos e com a suspensão de todas as aquisições de livros.

A fusão de bibliotecas e as alterações realizadas no SIBE/CIBEC acabaram por descaracterizar a biblioteca original do INEP, comprometendo a qualidade das informações disponibilizadas, especialmente no que se refere à especificidade do acervo. Ao longo de sua trajetória, o CIBEC passou por momentos críticos, chegando a ter a sua extinção e a doação de todo o seu acervo informacional ao governo do Distrito Federal publicadas no Diário Oficial da União, no início do governo Collor.

Todavia, o desmantelamento do CIBEC, que chegou a ser o principal centro de documentação sobre educação no Brasil, não se concretizou. Em 1992, no âmbito do INEP, ao CIBEC foi atribuída a responsabilidade pela implementação do Projeto Centro de Referências Sobre Inovações e Experimentos Educacionais (CRIE). Concebido para operar um grande e moderno centro de informações, esse projeto visava à constituição de um sistema de seleção, organização e disseminação de informações, experimentos e inovações educacionais,

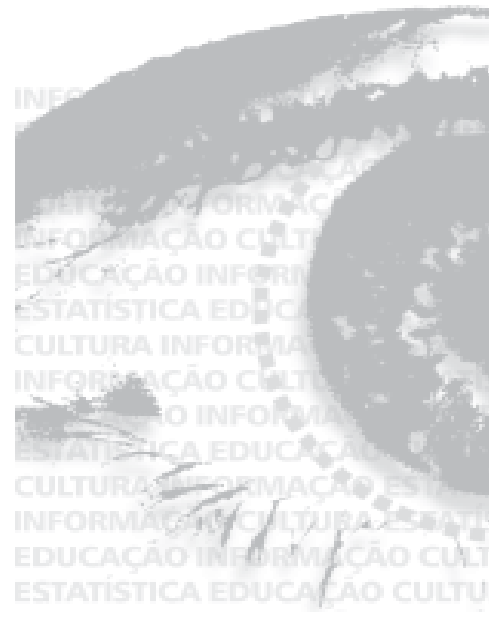


que contribuíssem para a formulação de políticas públicas e oferecessem subsídios para a melhoria da qualidade da Educação Básica, em microperspectiva. Além disso, desenvolveria mecanismos e instrumentos de captação, tratamento e divulgação de dados dispersos em acervos fragmentados em todo o território nacional.

Com o CRIE, pretendeu-se equacionar problemas de dimensões muito amplas, que exigiam, num prazo relativamente curto, uma proposta técnica correspondente. O próprio conceito de inovação envolvia diferentes interpretações, nem sempre compatíveis entre si. Assim, em 1995, quando a política educacional brasileira passou por uma profunda mudança em sua estrutura político-institucional, o MEC conferiu ao INEP as atividades de produção e disseminação de informações primárias referentes à Educação Básica e Superior, além da realização de censos e estatísticas para todos esses níveis de ensino. Nesse contexto, uma reavaliação do CRIE constatou a existência de um descompasso entre seus objetivos e as atividades realizadas, tanto por inadequação ao redesenho institucional pelo qual o MEC e o INEP passavam, quanto pela escassez de recursos materiais $e$ humanos, resultante dos rumos da gestão do setor público, nos anos anteriores.

A avaliação do CRIE indicou a necessidade de articulação entre as informações produzidas pelo Projeto e demais áreas de atuação do INEP com vistas a permitir a racionalização de custos e serviços relativos aos processos de coleta e disseminação de informações, considerando-se, ainda, que a própria metodologia de coleta era deficiente no que se referia aos critérios técnico-científicos.

Nessas circunstâncias, a reformulação do CRIE revelou-se indispensável. Em juIho de 1996, um novo projeto - o do Centro de Referência e Difusão em Educação (CRED) - foi idealizado. O CRED foi concebido como um instrumento centralizador de informações produzidas em áreas prioritárias para a formulação de políticas educacionais, estruturando-se para: (i) coletar, analisar, produzir, armazenar, disseminar e realizar o intercâmbio de informações sobre educação no Brasil e no exterior; (ii) organizar e manter acervos bibliográficos, históricos, arquivístivos, cadastrais e estatísticos e (iii) ampliar a capacidade gerencial e técnica dos usuários. Assim, o CRED incorporaria parte das atribuições do CRIE e resgataria as funções originais do CIBEC. Tal concepção, contudo, era demasiado abrangente e sua implantação deveria ser concomitante à elaboração de uma nova estrutura e missão do INEP. Essa situação acabou por comprometer a proposta de ação do Projeto.

\section{O CIBEC na Perspectiva do Atual INEP}

O INEP foi transformado em autarquia federal pela Lei $n$ ㅇ 9.448, de 14 de fevereiro de 1997. Essa alteração institucional fazia parte do quadro de reorientação da política educacional brasileira, promovida pelo governo Fernando Henrique Cardoso. Diante da necessidade de vincular suas ações às políticas educacionais, o INEP fortaleceu alianças, tanto internas quanto externas ao MEC, e investiu em informação, tecnologia, avaliação e desenvolveu um programa de mobilização de recursos humanos, materiais e financeiros que garantissem patamares constantes de qualidade das informações educacionais.

O INEP, tal como está estruturado atualmente, desenvolve e subsidia ações na área educacional por meio de produtos informacionais de base primária e secundária. Desta forma, procura tornar acessível aos agentes decisórios, aos sistemas de ensino e à sociedade dados confiáveis referentes à extensão e à qualidade do ensino ministrado no país, compreendendo as seguintes atividades: (i) investimento na produção de informações primárias a partir das sistemáticas de avaliação e dos novos instrumentos de ação; (ii) realização anual de pesquisas censitárias nacionais sobre os diversos níveis do sistema educacional e sobre o sistema de financiamento da educação; (iii) disseminação das informações obtidas; (iv) articulação dessas informações com as fontes secundárias e sua disseminação.

Além de realizar as pesquisas descritas anteriormente e de manter a base de dados correspondente, disseminando as informações nela contidas, o INEP promove a discussão de temas educacionais nos periódicos que edita e facilita o acesso de usuários a outras fontes de produção do saber 
educacional. Dessa maneira, o INEP contribui para o fortalecimento da capacidade gerencial das escolas, das secretarias estaduais e municipais de educação e do próprio MEC.

Como parte da reestruturação institucional do INEP e acompanhando a evolução do uso de recursos tecnológicos, teve início, em julho de 1997, o Programa de Tratamento e Disseminação de Informação Educacional, cuja instância executora é o Centro de Informações e Biblioteca em Educação, que manteve a sigla CIBEC. Este Programa visa a disseminar as informações primárias produzidas pelo INEP e por fontes secundárias, segundo princípios de democratização da informação, transparência das ações governamentais e busca da melhoria da qualidade da educação brasileira.

No momento da reformulação do CIBEC, grande parte dos acervos cadastrais, estatísticos, históricos, arquivísticos e bibliográficos, na área educacional, eram desconhecidos ou encontravam-se dispersos pelo território nacional. Com o Programa de Tratamento e Disseminação de Informação Educacional, esses problemas são minimizados tornando as informações acessíveis a administradores educacionais, pesquisadores, professores e ao público em geral.

\section{0 novo Centro de Informações e Biblioteca em Educação (CIBEC)}

A reestruturação do CIBEC permitiu a ampliação de seus serviços e produtos e criou condições de atendimento local e remoto por meio de equipamentos multimídia para acesso individualizado, de uma equipe multidisciplinar de facilitadores e de um programa de treinamento para a utilização de seus produtos. Para que essa estrutura de atendimento ao usuário fosse implementada, o CIBEC passou por uma profunda reforma do seu espaço físico, consolidando um local diferenciado e interativo para a disseminação de informações educacionais.

O CIBEC tem um caráter dinâmico em relação às suas ações, baseado em avaliações constantes sobre a qualidade do atendimento e dos serviços e produtos ofereci- dos, bem como da informação em si. Novos produtos estarão permanentemente sendo desenvolvidos e disseminados com vistas a responder aos imperativos de promoção do conhecimento a partir do amplo acesso a bases fidedignas e atualizadas de informações na área educacional.

Dessa forma, com a expansão dos canais de divulgação e uso orientado dos recursos informacionais, busca-se contribuir para a democratização do acesso à informação e para a melhoria da qualidade da educação no Brasil.

\section{PRODUTOS}

Publicações do INEP - O INEP mantém uma linha editorial própria que inclui a Revista Brasileira de Estudos Pedagógicos (RBEP) (quadrimestral), a coleção Em Aberto (trimestral) e a Série Documental, formada por um conjunto de séries temáticas, caracterizadas pela diversidade quanto ao público, periodicidade e referenciação. Todos esses produtos são distribuídos mediante solicitação.

Biblioteca Virtual de Educação (BVE) A BVE, que já pode ser acessada via Internet, é um catálogo de sites nacionais e internacionais na área de educação, selecionados e organizados em quatro grandes categorias, divididas em subcategorias: 1) Avaliação Educacional - da Educação Básica e da Educação Superior; 2) Estatística Educacional - da Educação Básica e da Educação Superior; 3) Outros Temas Currículos, Didática, Educação a Distância, Educação de Jovens e Adultos, Educação e Trabalho, Financiamento da Educação, Gestão do Ensino, História da Educação, Políticas Governamentais, entre outros e 4) Tipologia da Informação - Bases de Dados, Bibliotecas, Cursos de Pós-Graduação, Legislação, Organizações Governamentais, entre outras.

Perfil Municipal de Educação Básica (PMEB) - O PMEB também já pode ser acessado via Internet. Consiste num aplicativo que reúne variáveis socioeconômicas e educacionais sobre o conjunto de municípios brasileiros, regiões metropolitanas, estados e o Distrito Federal, grandes regiões e o território nacional.

Programa de Legislação Educacional Integrada (PROLEI) - Indexa a legislação educacional brasileira no âmbito federal,

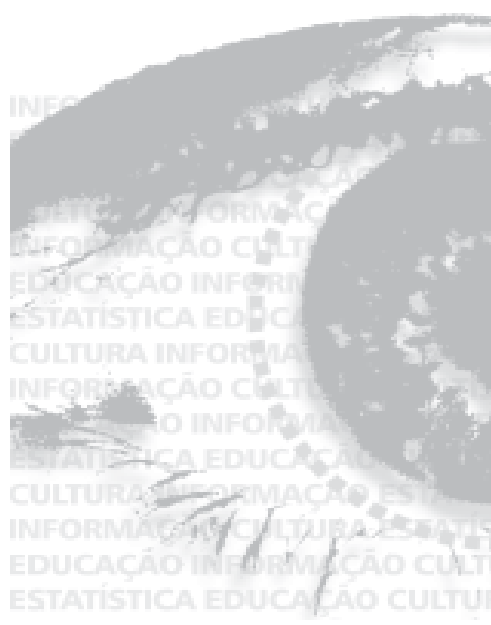


publicada a partir de dezembro de 1996, estabelecendo a correlação entre as normas jurídicas.

Thesaurus Brasileiro de Educação (BRASED) - É uma linguagem documental que utiliza vocabulário controlado, podendo ser utilizada como mecanismo de localização de documentos e de indexação. O BRASED será disseminado via Internet, incluindo-se um sistema de "reuniões virtuais" que permitam a sua permanente atualização e disponibilizará, para consulta, uma Lista Alfabética de Termos e uma Lista de Termos Candidatos.

Bibliografia Brasileira de Educação (BBE) - A BBE, seguindo os pólos de excelência do INEP na área educacional avaliação e estatística -, indexará artigos, estudos, ensaios e livros nas diferentes temáticas educacionais. As referências bibliográficas serão disponibilizadas na Internet. A base relativa aos anos posteriores a 1991, com atualização on-line e módulo de pesquisa, estará disponível no final de 1998.

Sumários Correntes - Serão editados mensalmente, com base no processo de seleção do CIBEC, disseminados em forma impressa e disponibilizados para consulta local.

Teses e Dissertações - O CIBEC dispõe de um acervo de teses produzidas no âmbito das faculdades de educação de todo o país, que passarão a ser divulgadas periodicamente através da $R B E P$, a partir deste número (191).

Lançamentos Editoriais em Educação - Este serviço consiste numa seleção de títulos na área educacional, publicados pelas diversas editoras do país e do exterior, a serem divulgados pela RBEP a partir deste número (191).

Horário de funcionamento - O CIBEC funciona de segunda a sexta-feira, no período de $8 \mathrm{~h}$. às $20 \mathrm{~h}$.

Empréstimos - Os itens que integram o acervo do CIBEC estão disponíveis para consulta local, mas não são cedidos em empréstimo. Cópias podem ser solicitadas, respeitados os direitos autorais.

Biblioteca Automatizada - A Biblioteca está sendo totalmente automatizada, inclusive no que se refere à indexação dos acervos bibliográfico, histórico, audiovisual e de obras raras, produzidos em diversas mídias e com módulo de pesquisa via Internet.

Acervo Bibliográfico - O acervo do CIBEC passou por uma ampla avaliação com o objetivo de alcançar maior especialização nas áreas de atuação do INEP - avaliação e estatísticas educacionais - , sem desconsiderar, entretanto, a amplitude e a complexidade da temática educação. Desta forma, foi empreendida uma atualização de um conjunto de coleções de periódicos e retomadas as aquisições de publicações de destaque e relevância na área.

COMUT - O CIBEC dispõe deste serviço on-line que pode ser solicitado por todos os usuários.

Disseminação Seletiva da Informação O Centro oferece serviços de disseminação seletiva da informação em bases secundárias internacionais e nacionais, com atualização periódica. Encontram-se disponíveis para consulta local o Current Contents e o Social Sciences Citation Index, desenvolvidos pelo Institute of Scientific Information (ISI). O Current Contents é elaborado a partir de uma base de dados de aproximadamente 2.500 periódicos, selecionados segundo rigorosos critérios, que dissemina referências bibliográficas e respectivos resumos e oferece um módulo interativo de pesquisa. O Social Sciences Citation Index é uma base de dados semelhante à do Current Contents, que oferece, ainda, indicações de citações, tanto de quem é citado por determinado autor e em que obra, quanto do número de vezes em que esse autor é citado. A busca no Index pode ser feita por assunto, autor, título de periódico ou país. Podem ser utilizados também recursos adicionais para o refinamento da pesquisa realizada.

Pesquisas Bibliográficas - O CIBEC dispõe de estrutura para a realização de pesquisas em suas bases de dados, tanto estatísticas quanto bibliográficas. Estas podem ser feitas localmente, com a orientação de facilitadores, caso necessário, ou solicitadas por e-mail, fax ou correio.

Obras raras - Trata-se de uma coleção de aproximadamente 1.600 obras raras e especiais sobre educação e outros temas de caráter geral na área de ciências sociais. Esse valioso acervo, totalmente restaurado, é acessível, parcialmente, em exposições 
temáticas e cópias fiéis produzidas pelo CIBEC.

Treinamento - O CIBEC proporciona treinamento constante a seus usuários na utilização de seus serviços e produtos. Os programas podem ser solicitados por instituições ou indivíduos. Além disso, os usuários não familiarizados com as tecnologias e técnicas utilizadas podem contar com facilitadores com formação multidisciplinar.

\section{PARTICIPAÇÃO EM REDES}

\section{RED LATINOAMERICANA DE INFORMACIÓNY DOCUMENTACÍON EN EDUCACIÓN (REDUC)}

Criada em 1977, a REDUC é um sistema dedicado à preservação da memória da produção latino-americana em educação. Adota um modelo cooperativo, tendo como Coordenador o Centro de Investigación y Desarollo Educativo (CIDE), situado em Santiago, Chile. Conta, atualmente, com 22 centros filiados em 19 países, incluindo-se a maior parte dos países da América Latina, os Estados Unidos e o Canadá.

A REDUC acumula mais de 15 mil registros de pesquisas finalizadas ou em andamento, experiências e documentos de interesse originados na América Latina ou que a ela se refiram, apresentados nas seguintes modalidades de produtos: Resumos Analíticos de Educação (RAEs), Resumos Analíticos Monotemáticos em Educação (RAM), Estados da Arte, Bibliografias de Pesquisas e Ensaios (BIE).

O INEP participa da REDUC como Centro Associado, ao lado da Fundação Carlos Chagas. Por essa razão, o INEP estabeleceu, como norma aos colaboradores dos periódicos de sua linha editorial, a elaboração de resumos no formato REDUC, disseminados pela própria Rede e pela $B B E$.

\section{REDE ANTARES}

O INEP faz parte da Rede Antares, na categoria de Provedor de Produtos e Serviços de Informação, ampliando, desta forma, as possibilidades de acesso, via Internet, às informações disponibilizadas pelo Instituto.

\section{COMITÊ DOS PRODUTORES DA} INFORMAÇÃO EDUCACIONAL (COMPED)

O COMPED, criado em 12 de fevereiro de 1997, é formado pelo INEP e as seguintes instituições: Associação Brasileira de Editoras Universitárias (ABEU), Associação Nacional de Pós-Graduação e Pesquisa em Educação (ANPED), Associação Nacional de Profissionais de Administração da Educação (ANPAE), Centro de Estudos e Pesquisas em Educação, Cultura e Ação Comunitária (CENPEC), Conselho Nacional de Desenvolvimento Científico e Tecnológico (CNPq), Conselho Nacional de Secretários de Educação (CONSED), Coordenação de Aperfeiçoamento de Pessoal de Nível Superior (CAPES), Fundação Carlos Chagas (FCC), Fundação Cesgranrio, Instituto Brasileiro de Informações em Ciência e Tecnologia (IBICT), Instituto de Pesquisa Econômica Aplicada (IPEA), Programa das Nações Unidas para o Desenvolvimento (PNUD) e União Nacional dos Dirigentes Municipais de Educação (UNDIME).

A finalidade do COMPED é desenvolver um sistema articulado de disseminação de informações educacionais, proceder ao mapeamento de lacunas informacionais e promover a sua superação. Neste sentido, já estão sendo desenvolvidos estados da arte sobre os seguintes temas: alfabetização, educação de crianças de 0 a 6 anos, formação de professores, ensino superior, avaliação educacional no âmbito do ensino básico e políticas e gestão da educação.

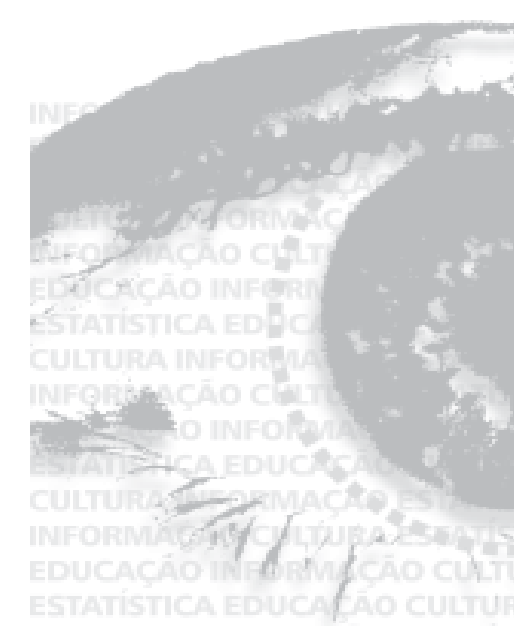




\section{Resumo}

Descreve a evolução do Centro de Informações e Biblioteca em Educação (CIBEC), desde a biblioteca constituída a partir da criação do Instituto Nacional de Estudos e Pesquisas Educacionais (INEP), e o papel que Ihe corresponde a partir da reformulação do INEP pela Lei $n^{\circ}$ 9.448/97. Apresenta os múltiplos produtos e serviços do INEP disponibilizados pelo CIBEC, indicando as formas de acesso público aos mesmos, diretamente nas instalações inauguradas em novembro de 1998, no andar térreo do Ministério da Educação e do Desporto (MEC), ou por meio virtual.

Palavras-Chave: Centro de Informações e Biblioteca em Educação, histórico.

\section{Abstract}

This work portrays the development of the Information Center and Library in Education (CIBEC) since it was established, after the creation of the National Institute of Educational Studies and Research and the role CIBEC plays after INEP's reformulation by the Law $N^{\circ}$ 9.448/977. The work also presents various INEP's products and services made available by $C I B E C$, indicating public ways to access them in the recent facilities inauguration (November 1998 ) or by virtual mode. CIBEC is located in the ground floor of the Ministry of Education and Sports (MEC).

Key-Words: Information Center and Library in Education, historical. 